\title{
Effects of exercise on mature or precursor brain-derived neurotrophic factor pathways in ovariectomized rats
}

\author{
JOON-KI PARK ${ }^{1}$, YOUNG-PYO HONG ${ }^{2}$ and SAM-JUN LEE ${ }^{3}$ \\ ${ }^{1}$ Division of Exercise and Health Science, College of Arts and Physical Education, Incheon National University, \\ Incheon 22012; ${ }^{2}$ Health Education Laboratory, Department of Physical Education, \\ Korea National Sport University, Seoul 05541; ${ }^{3}$ Department of Physical Education, College of Health, \\ Social Welfare and Education, Tong Myong University, Busan 48520, Republic of Korea
}

Received March 30, 2016; Accepted February 27, 2017

DOI: $10.3892 / \mathrm{mmr} .2017 .6614$

\begin{abstract}
Ovariectomy (OVX) is a method used to block estrogen in female rats that induces hippocampal dysfunction and affects brain-derived neurotrophic factor (BDNF) pathways. The majority of previous studies investigating OVX focused on BDNF expression in the hippocampus and cognitive function. The present study focused on the pathways of each BDNF type, precursor (proBDNF) and mature (mBDNF), and the effects of regular exercise in the hippocampus of ovariectomized rats. Female Sprague-Dawely rats were used and OVX surgery was performed. After 1 week of recovery from surgery, two groups of rats that received OVX surgery were subjected to regular treadmill exercise for 8 weeks. The results of protein levels by western blotting indicated that the expression of proBDNF, p75 neurotrophin receptor (p75NTR) and c-Jun N-terminal protein kinase (JNK) was increased, and mBDNF, tropomyosin-related kinase B (TrkB) and nuclear factor- $\kappa \mathrm{B}$ expression was significantly reduced in the OVX control group compared with the sham control group $\mathrm{SC}(\mathrm{P}<0.05)$. Thus, the survival pathway by mBDNF was impaired and the pro-apoptotic response was activated by increased JNK expression due to proBDNF-p75NTR binding in the hippocampus of ovariectomized rats. By contrast, exercise reduced activation of the pro-apoptotic response and increased mBDNF-TrkB expression in the hippocampus of ovariectomized rats. Thus, regular exercise may increase the activation of survival pathways via mBDNF and reducing the activation of the pro-apoptotic pathway of proBDNF in the hippocampus of ovariectomized rats.
\end{abstract}

Correspondence to: Professor Sam-Jun Lee, Department of Physical Education, College of Health, Social Welfare and Education, Tong Myong University, 179 Sinseonno, Busan 48520, Republic of Korea

E-mail: anada23@tu.ac.kr

Key words: ovariectomy, estrogen, exercise, brain-derived neurotrophic factor, hippocampus, neurotrophin

\section{Introduction}

An animal model of ovariectomy (OVX) previously demonstrated that OVX induced blockade of estrogen (1). The resulting estrogen deficiency has been reported to be closely associated with brain dysfunction in the hippocampus (2). After binding to estrogen receptor $\alpha(\mathrm{ER} \alpha)$ or $\beta$ $(\mathrm{ER} \beta)$, estrogen promotes mitogen-activated protein-kinase (MAPK), phosphatidylinositol 3-kinase (PI3K) and phospholipase $\mathrm{C} \gamma(\mathrm{PLC} \gamma)$ pathways (3), which influence synaptic formation, neuronal plasticity, cognition and neuroprotection in neurodegenerative diseases (4-7). In addition, estrogen has an important role in the synthesis and expression of brain-derived neurotrophic factor (BDNF), which regulates neuronal function in the hippocampus (8). BDNF exists as two forms in tissues: Precursor BDNF (proBDNF) and mature BDNF (mBDNF). mBDNF is synthesized from proBDNF following cleavage by tissue plasminogen activator (tPA) (9). In general, mBDNF acts by binding to the tropomyosin-related kinase B (TrkB) receptor in the MAPK and PI3K-Akt pathways (10-14), which has positive effects for neuronal proliferation, differentiation and the development of synapses associated with hippocampal function $(15,16)$. ProBDNF also acts positively for neuronal function by binding to the p75 neurotrophin receptor (p75NTR). However, the proBDNF-p75NTR pathway also includes pro-apoptotic activation (10-14). BDNF expression is reduced by OVX, which induces an estrogen block $(17,18)$. In an animal model of OVX, BDNF was reduced markedly and hippocampal function declined (2). Previous studies investigating hippocampal function in OVX focused on mBDNF pathways alone $(17,18)$. Contrary to the previous OVX results, regular exercise has been previously demonstrated to have positive effects on brain function $(19,20)$, and affects the proliferation, differentiation and survival of neuronal cells, and aids neuronal plasticity by promoting neurotrophins (21). Exercise enhances BDNF expression by positively affecting tPA, which is associated with the synthesis of mBDNF in the hippocampus (22) and the TrkB pathway $(16,23,24)$. Chronic exercise has neuroprotective effects on hippocampal dysfunction, dementia and neurodegenerative diseases (25-28). In addition, exercise improves cognitive decline induced by OVX (1). The majority 
of previous studies regarding the effects of exercise on brain function by OVX focused on BDNF expression and cognition in the hippocampus (29-31). A limited number of studies have sought to determine the underlying mechanism of proBDNF and mBDNF pathways associated with hippocampal function in OVX. The present study investigated whether regular exercise increased mBDNF synthesis from proBDNF in the OVX rat model, and determined the underlying molecular mechanisms of proBDNF and mBDNF signaling pathways.

\section{Materials and methods}

Animals. Female, 6-week-old Sprague-Dawley rats $(\mathrm{n}=50$; 160-200 g; Samtako Bio Korea Co., Ltd., Republic of Korea) were adapted to the laboratory environment (temperature, $22 \pm 1^{\circ} \mathrm{C}$; relative humidity, $55 \pm 3 \%$; 12 -h light/12-h dark photoperiod) for 2 weeks. All rats were housed in pairs, given free access to water and fed a standard chow diet (protein, 21\%; fat, $5 \%$; nitrogen-free extract, $55 \%$; fiber, $4 \%$; adequate mineral and vitamin content). Studies were performed in accordance with Chung-Nam University standards for the Care and Use of Laboratory Animals (publication no. CNU-00203). Rats were allocated to the following groups: Sham control group (SC; $n=10)$; OVX control group (OC; $n=10)$; OVX low-exercise group (OLE; $\mathrm{n}=10)$; and OVX moderate-exercise group (OME; $\mathrm{n}=10)$.

Ovariectomy. Following anesthetization using ketamine/xylazine ( $8 \mathrm{mg} / \mathrm{kg}$ body weight), the dorsal midlumbar area between the first and third lumbar was shaved and the midline was incised. A single 5.5-10 mm long incision was made in the muscle wall on the right and left sides approximately one-third of the distance between the spinal cord and the ventral midline. The ovary was exteriorized through the muscle wall and removed. In the SC group, the ovary was exteriorized but not removed.

Exercise protocol. After a 1-week recovery from surgery, rats in the OLE and OME groups were subjected to treadmill exercise for 8 weeks, 5 days a week. The speed of treadmill exercise was $8 \mathrm{~m} / \mathrm{min}$ (grade $0 \%$ ) in weeks $1-4$ and $10 \mathrm{~m} / \mathrm{min}$ (grade 0\%) in weeks 5-8 for the OLE group, and $12 \mathrm{~m} / \mathrm{min}$ in weeks $1-4$ and $18 \mathrm{~m} / \mathrm{min}$ (grade $0 \%$ ) in weeks $5-8$ for the OME group (32). The duration was gradually increased from 30 to $60 \mathrm{~min}$. OLE and OME groups were subjected to $30 \mathrm{~min}$ for in weeks 1 and 2, $40 \mathrm{~min}$ in weeks 3 and 4, $50 \mathrm{~min}$ in weeks 5 and 6 , and $60 \mathrm{~min}$ in weeks 7 and 8 . The non-exercise groups, including SC and OC, were exposed to environmental stress similar to that experienced with treadmill use, including noise and vibration, and restricted food and water during treadmill exercise. To minimize the stress of exercise, treadmill exercise was performed without external stimuli and electronic shock.

Tissue preparation. Upon completion of the 8-week exercise program, the rats were anesthetized at $48 \mathrm{~h}$ after the final exercise session by intraperitoneal injection of xylazine $(8 \mathrm{mg} / \mathrm{kg})$ and ketamine $(40 \mathrm{mg} / \mathrm{kg})$. For protein analyses, brains of rats from each group were extracted and the hippocampus was dissected and stored at $-80^{\circ} \mathrm{C}$.
Western blotting. To prepare protein for western blotting, each hippocampus was crushed in a solution containing $150 \mathrm{mM}$ $\mathrm{NaCl}, 5 \mathrm{mM}$ EDTA, 50 mM Tris- $\mathrm{HCl}$ (pH 8.0), 1\% NP-40, $1 \mathrm{mM}$ aprotinin, $0.1 \mathrm{mM}$ leupeptin and $1 \mathrm{mM}$ pepstatin, and centrifuged at $15,294 \times \mathrm{g}$ for $15 \mathrm{~min}$ at $4^{\circ} \mathrm{C}$. Proteins were quantified by a Bradford assay and $30 \mu \mathrm{g}$ was loaded onto a $10 \%$ gel, subjected to SDS-PAGE and transferred to a polyvinylidene difluoride membrane (EMD Millipore, Billerica, MA, USA). The membrane was blocked in TBS containing $0.001 \%$ Tween-20 (TBS-T) and 5\% bovine serum albumin (Bovogen Biologicals Ltd., Victoria, Australia) at $4^{\circ} \mathrm{C}$ for $90 \mathrm{~min}$. After washing, the membrane was incubated overnight at $4^{\circ} \mathrm{C}$ with the following primary antibodies: Rabbit anti-GAPDH (1:1,000; catalog no. ABS16; EMD Millipore, Billerica, MA, USA), rabbit anti-proBDNF (1:1,000; catalog no. sc-546; Santa Cruz Biotechnology, Inc., Dallas, TX, USA), rabbit anti-mBDNF (1:1,000; catalog no. sc-546; Santa Cruz Biotechnology, Inc.), rabbit anti-tPA (1:1,000; catalog no. sc-15346; Santa Cruz Biotechnology, Inc.), rabbit anti-TrkB (1:1,000; catalog no. sc-119; Santa Cruz Biotechnology, Inc.), rabbit anti-p75NTR (1:1,000; catalog no. 4201S; Cell Signaling Technology, Inc., Danvers, MA, USA), rabbit anti-total (t)-JNK (1:1,000; catalog no. sc-571; Cell Signaling Technology, Inc.), rabbit anti-phosphorylated (p)-JNK (1:1,000; catalog no. sc-135642; Cell Signaling Technology, Inc.) and rabbit anti-nuclear factor- $\kappa \mathrm{B}(\mathrm{NF}-\kappa \mathrm{B} ; 1: 1,000$; catalog no. 3031S; Cell Signaling Technology, Inc.). Subsequently, membranes were washed 3 times with TBS-T for $10 \mathrm{~min}$ and incubated with a goat anti-rabbit IgG secondary antibody conjugated to alkaline phosphatase $(1: 2,000$; catalog no. sc-2007; Santa Cruz Biotechnology, Inc.) for $1 \mathrm{~h}$ at room temperature. The membrane was washed 3 times with TBS-T for $10 \mathrm{~min}$. Membranes were exposed to Luminata ${ }^{\mathrm{TM}}$ (EMD Millipore, Billerica, MA, USA) and protein bands were imaged using a Kodak Image Station 440CF (Kodak, Rochester, NY, USA) and were quantified using Kodak ID version 3.5 densitometry software (Kodak). Membranes were stripped with stripping buffer [20 ml 10\% SDS, $12.5 \mathrm{ml} 0.5 \mathrm{M}$ Tris HCL (pH 6.8), $67.5 \mathrm{ml}$ ultra pure water, $0.8 \mathrm{ml}$ beta-mercaptoethanol] for $45 \mathrm{~min}$ at $50^{\circ} \mathrm{C}$ and washed 5 times with TBS-T for $10 \mathrm{~min}$. Following stripping, membranes were blocked and re-probed with the appropriate primary antibody 3-4 times. This was performed at least twice, and the results demonstrate blots from one representative experiment.

Statistical analysis. All data was analyzed using SPSS software version 16.0 (SPSS, Inc., Chicago, IL, USA) by one-way analysis of variance followed by Tukey's post hoc test to compare among the experimental groups. Results are presented as the mean + standard deviation. $\mathrm{P}<0.05$ was considered to indicate a statistically significant difference.

\section{Results}

Effects of exercise on $m B D N F$, TrkB and $t P A$ in the hippocampus. The OC group exhibited reduced mBDNF, TrkB and tPA protein expression in the hippocampus compared with the SC group (Fig. 1). By contrast, in the OLE and OME groups, significantly increased mBDNF, TrkB and tPA protein expression was observed in the hippocampus compared with 
A
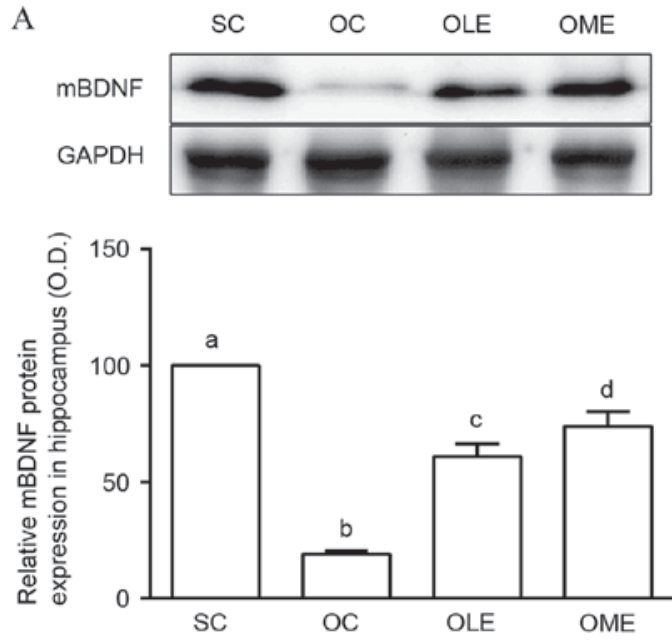

C
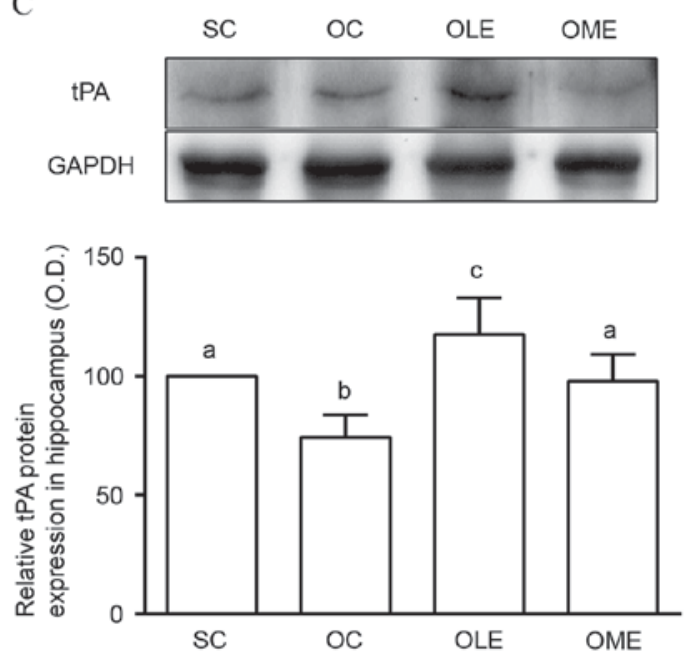

B
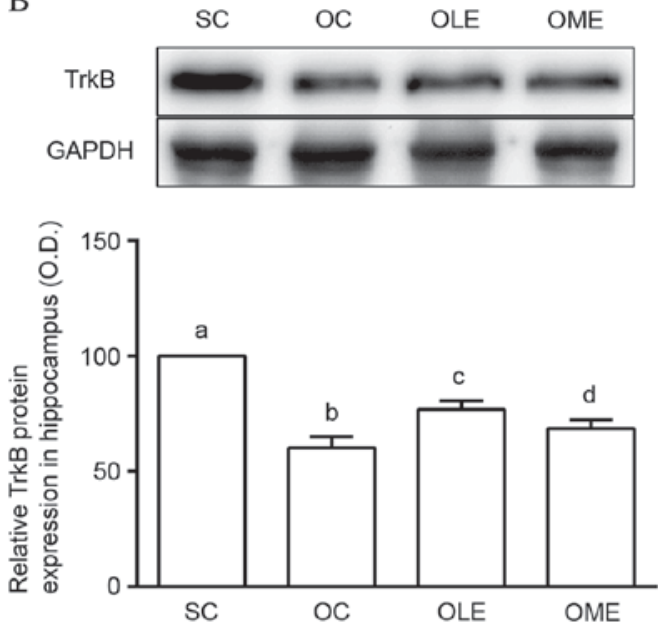

Figure 1. Expression of (A) mBDNF, (B) TrkB and (C) tPA protein in the hippocampus of control and OVX rats. Results are presented as the mean + standard deviation. Different letters represent significant differences compared with the other groups $(\mathrm{P}<0.05)$. $\mathrm{mBDNF}$, mature brain-derived neurotrophic factor; TrkB, tropomyosin-related kinase B; tPA, tissue plasminogen activator; OVX, ovariectomy; SC, sham control; OC, OVX control; OLE, OVX + low-exercise; OME, OVX + moderate-exercise; O.D., optical density.

the OC group (Fig. 1). Exercise intensity differences were also apparent as expression levels were significantly different between the OLE and OME groups (Fig. 1).

Effects of exercise on proBDNF and p75NTR in hippocampus. The OC group exhibited significantly increased proBDNF and p75NTR protein expression in the hippocampus compared with the SC group (Fig. 2). Furthermore, the OLE and OME groups had reduced proBDNF and p75NTR protein expression in the hippocampus compared with the OC group (Fig. 2). There were significant differences in exercise intensity between the OLE and OME groups ( $\mathrm{P}<0.05$; Fig. 2).

Effects of exercise on $t-J N K, p-J N K$, and $N F-\kappa B$ in the hippocampus. A significantly increased p-JNK/t-JNK ratio and reduced $\mathrm{NF}-\kappa \mathrm{B}$ protein expression was observed in the hippocampus of OC rats compared with the SC group (Fig. 3). In addition, a reduced $\mathrm{p}-\mathrm{JNK} / \mathrm{t}-\mathrm{JNK}$ ratio and increased $\mathrm{NF}-\kappa \mathrm{B}$ protein expression was observed in the hippocampus of OLE and OME groups, compared with the OC group (Fig. 3). There were significant differences in $\mathrm{NF}-\kappa \mathrm{B}$ expression between the OLE and OME groups ( $\mathrm{P}<0.05$; Fig. 3$)$.

\section{Discussion}

The present study investigated the effects of exercise on BDNF pathways in the hippocampus of ovariectomized rats and demonstrated that $\mathrm{PA}, \mathrm{mBDNF}$ and $\mathrm{NF}-\kappa \mathrm{B}$ protein expression was reduced, and the proBDNF, p75NTR and p-JNK level was increased by OVX compared with sham rats. The observed alterations in protein expression were reversed by regular exercise.

As a sex hormone, estrogen regulates diverse processes, including bone mineral density and calcium intake. Estrogen also influences intracellular pathways, synaptic structure and physiological functions of neurons in several brain regions (4-6), and regulates neurotrophin expression and has neuroprotective effects against diseases (2). In the hippocampus, estrogen is particularly important in enhanced cognitive functions by supporting neurogenesis and synaptic 
A
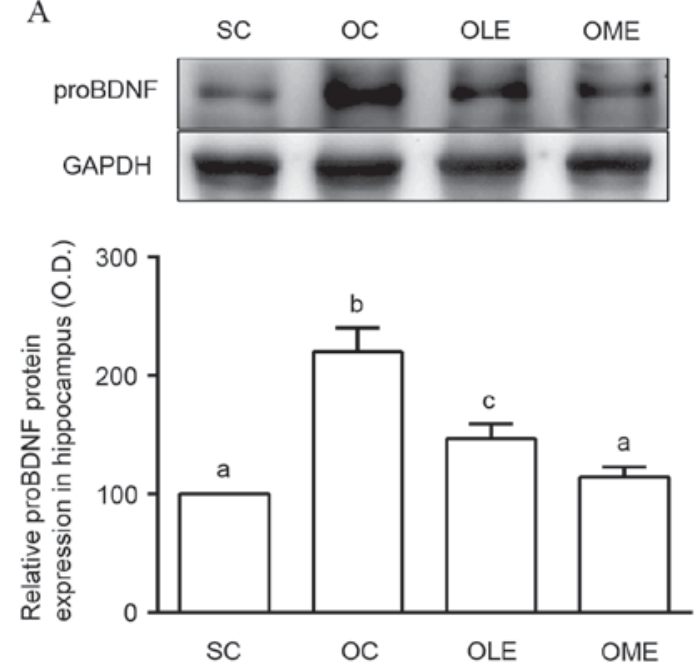

B
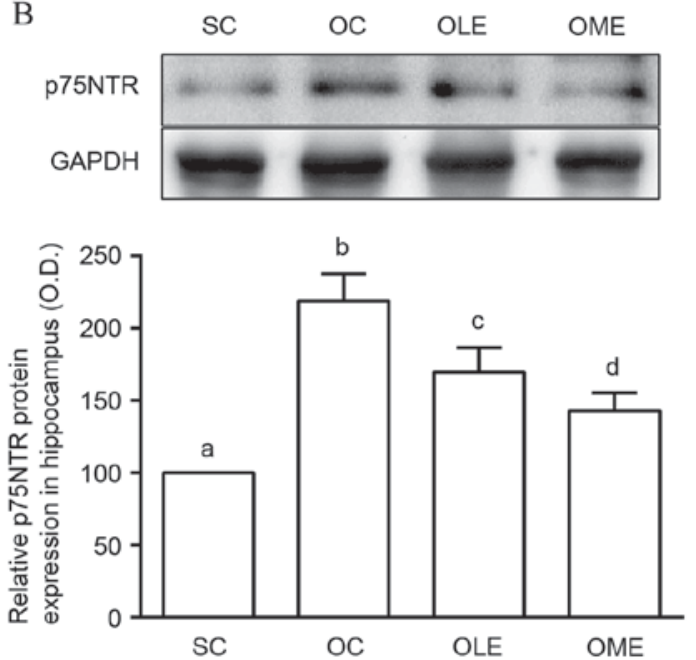

Figure 2. Expression of (A) proBDNF and (B) p75NTR protein in the hippocampus of control and OVX rats. Results are presented as the mean + standard deviation. Different letters represent significant differences compared with the other groups $(\mathrm{P}<0.05)$. proBDNF, precursor brain-derived neurotrophic factor; p75NTR, p75 neurotrophin receptor; OVX, ovariectomy; SC, sham control; OC, OVX control; OLE, OVX + low-exercise; OME, OVX + moderate-exercise; O.D., optical density.

A
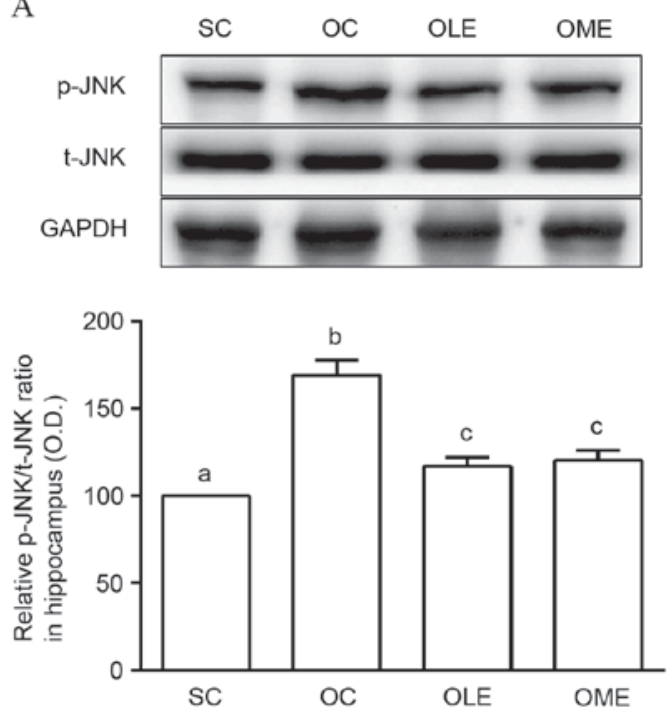

B
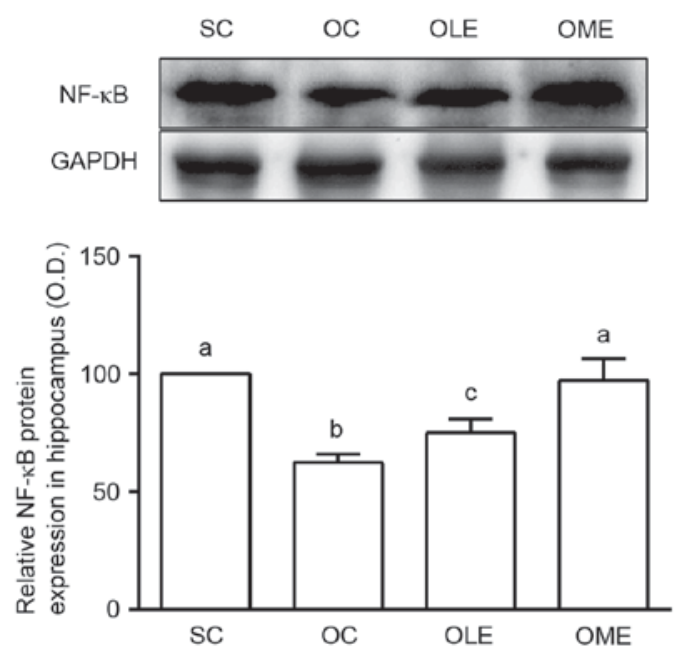

Figure 3. Expression of (A) p-JNK/t-JNK and (B) NF- $\mathrm{KB}$ protein in the hippocampus of control and OVX rats. Results are presented as the mean + standard deviation. Different letters represent significant differences compared with the other groups $(\mathrm{P}<0.05)$. JNK, c-Jun N-terminal protein kinase; p-JNK, phos-

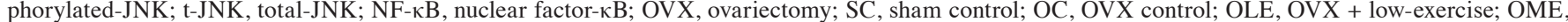
OVX + moderate-exercise; O.D., optical density.

plasticity, and protects hippocampal function from neurodegenerative disease $(2,7)$. Estrogen has direct effects in various pathways through binding to estrogen receptors (17) or indirect neuronal functions by regulating extrinsic factors, such as neurotrophins (33). In particular, estrogen is associated with BDNF synthesis and expression in the hippocampus (8). The interaction between estrogen and BDNF is crucial for hippocampal functions $(3,34)$.

BDNF released from neuronal cells exists in precursor and mature forms, and mBDNF is synthesized from proBDNF $(9,35,36)$. proBDNF is proteolytically cleaved intracellularly by enzymes, including furin and pro-protein convertase (37-39), or secreted as proBDNF and subsequently cleaved to create mBDNF by extracellular proteases, such as metalloproteases and plasmin (40). Plasmin is converted from plasminogen by tPA as a key regulator of BDNF synthesis, and has a crucial role in BDNF-dependent, late-phase, long-term potential associated with hippocampal plasticity (40). Various studies investigating the interaction between estrogen and BDNF in the hippocampus have demonstrated that BDNF expression is reduced by an estrogen deficit in ovariectomized rats $(17,18)$. In the present study, hippocampal mBDNF was significantly reduced in the OVX treatment group compared with SC rats, and tPA also was reduced by OVX. The results indicate that TPA may be involved in the reduced expression of mBDNF induced by OVX. mBDNF exerts its actions on 
neuronal structure, function and synaptic plasticity underlying memory and cognition (41-43) by activation of MAPK, PI3K-Akt, PLC $\gamma, \mathrm{Ca}^{2+} /$ calmodulin-dependent protein kinase and cAMP response element-binding protein pathways following interactions with TrkB $(23,44)$.

While proBDNF was originally considered to be a precursor with no inherent biological function, multiple reports have indicated that proBDNF is secreted from neurons and modulates synaptic functions through certain pathways by binding p75NTR $(45,46)$. The interaction between proBDNF and p75NTR aids neuronal functions through underlying pathways (46). proBDNF-p75NTR functions in prosurvival responses via the $\mathrm{NF}-\kappa \mathrm{B}$ cascade (13), and is also associated with long-term hippocampal depression (45) and induces pro-apoptotic responses (10-14) via the JNK cascade (47-49). In particular, apoptosis induced by proBDNF-p75NTR has been previously demonstrated in neurodegenerative diseases; Sierksma et al (50) noted that hippocampal proBDNF was increased significantly in an animal model of Alzheimer's disease.

In the present study, the OVX group exhibited significantly higher proBDNF, p75NTR and JNK, associated with pro-apoptotic responses, compared with the SC group. Conversely, $\mathrm{NF}-\kappa \mathrm{B}$ was reduced by OVX. As a result, we hypothesize that the pro-apoptotic response of JNK underlying the proBDNF pathway functions in hippocampal dysfunctions induced by OVX. Estrogen deficit-associated hippocampal dysfunction may be associated with inhibition of the mBDNF pathway and the pro-apoptotic action of proBDNF. Exercise is beneficial for hippocampal functions $(19,20)$, and it is associated with increased mBDNF expression and signaling (22). In addition, exercise has a role in the improvement and protection of the hippocampus in neurodegenerative diseases (51). Jin et al (1) reported that exercise improved cognitive functions in a rat model of OVX. To investigate the effects of exercise on OVX, rats were subjected to treadmill exercise following OVX and it was observed that regular exercise improved BDNF pathways in the hippocampus, increasing the levels of mBDNF, TrkB and tPA, and reducing levels of proBDNF, p75NTR and JNK. These results suggested that exercise may suppress the pro-apoptotic response of the proBDNF-p75NTR pathway by increasing synthesis of $\mathrm{mBDNF}$ and activating tPA. Thus, exercise may enhance neuronal functions in the OVX rat brain.

The present study has several important implications. First, hippocampal dysfunction induced by OVX was caused by dysfunction of the mBDNF pathway and the pro-apoptotic response associated with the proBDNF pathway. In addition, it was demonstrated that pro-apoptotic action through the proBDNF-p75NTR cascade involved the JNK pathway. Furthermore, dysfunction of BDNF signaling was improved by exercise. Therefore, regular exercise may improve BDNF pathways in the hippocampus of OVX rats. These results may aid future studies investigating the effects of exercise on proBDNF and mBDNF pathways, in addition to hippocampal function, estrogen deficiency and menopause.

\section{Acknowledgements}

This work was supported by an Incheon National University Research Grant in 2013 (Incheon, Republic of South Korea).

\section{References}

1. Jin J, Jing H, Choi G, Oh MS, Ryu JH, Jeong JW, Huh Y and Park C: Voluntary exercise increases the new cell formation in the hippocampus of ovariectomized mice. Neurosci Lett 439: 260-263, 2008

2. Kiss A, Delattre AM, Pereira SI, Carolino RG, Szawka RE, Anselmo-Franci JA, Zanata SM and Ferraz AC: 17 $\beta$-estradiol replacement in young, adult and middle-aged female ovariectomized rats promotes improvement of spatial reference memory and an antidepressant effect and alters monoamines and BDNF levels in memory- and depression-related brain areas. Behav Brain Res 227: 100-108, 2012.

3. Scharfman HE and MacLusky NJ: Similarities between actions of estrogen and BDNF in the hippocampus: Coincidence or clue? Trends Neurosci 28: 79-85, 2005.

4. Woolley CS: Acute effects of estrogen on neuronal physiology. Annu Rev Pharmacol Toxicol 47: 657-680, 2007.

5. Srivastava DP: Two-step wiring plasticity-a mechanism for estrogen-induced rewiring of cortical circuits. J Steroid Biochem Mol Biol 131: 17-23, 2012.

6. Srivastava DP, Waters EM, Mermelstein PG, KramárEA, Shors TJ and Liu F: Rapid estrogen signaling in the brain: Implications for the fine-tuning of neuronal circuitry. J Neurosci 31: 16056-16063, 2011.

7. Henderson VW: Alzheimer's disease: Review of hormone therapy trials and implications for treatment and prevention after menopause. J Steroid Biochem Mol Biol 142: 99-106, 2014.

8. Solum DT and Handa RJ: Estrogen regulates the development of brain-derived neurotrophic factor $\mathrm{mRNA}$ and protein in the rat hippocampus. J Neurosci 22: 2650-2659, 2002.

9. Teng KK, Felice S, Kim T and Hempstead BL: Understanding proneurotrophin actions: Recent advances and challenges. Dev Neurobiol 70: 350-359, 2010.

10. Boyd JG and Gordon T: A dose-dependent facilitation and inhibition of peripheral nerve regeneration by brain-derived neurotrophic factor. Eur J Neurosci 15: 613-626, 2002.

11. Troy CM, Friedman JE and Friedman WJ: Mechanisms of p75-mediated death of hippocampal neurons. Role of caspases. J Biol Chem 277: 34295-34302, 2002.

12. Teng HK, Teng KK, Lee R, Wright S, Tevar S, Almeida RD, Kermani P, Torkin R, Chen ZY, Lee FS, et al: ProBDNF induces neuronal apoptosis via activation of a receptor complex of p75NTR and sortilin. J Neurosci 25: 5455-5463, 2005.

13. Reichardt LF: Neurotrophin-regulated signalling pathways. Philos Trans R Soc Lond B Biol Sci 361: 1545-1564, 2006.

14. Cunha C, Brambilla R and Thomas KL: A simple role for BDNF in learning and memory? Front Mol Neurosci 3: 1, 2010.

15. Lu Y, Christian K and Lu B: BDNF: A key regulator for protein synthesis-dependent LTP and long-term memory? Neurobiol Learn Mem 89: 312-323, 2008.

16. Yoshii A and Constantine-Paton M: Postsynaptic BDNF-TrkB signaling in synapse maturation, plasticity, and disease. Dev Neurobiol 70: 304-322, 2010.

17. Scharfman HE and MacLusky NJ: Estrogen and brain-derived neurotrophic factor (BDNF) in hippocampus: Complexity of steroid hormone-growth factor interactions in the adult CNS. Front Neuroendocrinol 27: 415-435, 2006.

18. Luine V and Frankfurt M: Interactions between estradiol, BDNF and dendritic spines in promoting memory. Neuroscience 239: 34-45, 2013.

19. Cotman CW and Berchtold NC: Exercise: A behavioral intervention to enhance brain health and plasticity. Trends Neurosci 25: 295-301, 2002.

20. Fabel K and Kempermann G: Physical activity and the regulation of neurogenesis in the adult and aging brain. Neuromolecular Med 10: 59-66, 2008.

21. Vivar C, Potter MC and van Praag H: All about running: Synaptic plasticity, growth factors and adult hippocampal neurogenesis. Curr Top Behav Neurosci 15: 189-210, 2013.

22. Sartori CR, Vieira AS, Ferrari EM, Langone F, Tongiorgi E and Parada CA: The antidepressive effect of the physical exercise correlates with increased levels of mature BDNF, and proBDNF proteolytic cleavage-related genes, p11 and tPA. Neuroscience 180: 9-18, 2011.

23. Chao MV, Rajagopal R and Lee FS: Neurotrophin signalling in health and disease. Clin Sci (Lond) 110: 167-173, 2006.

24. Minichiello L: TrkB signalling pathways in LTP and learning. Nat Rev Neurosci 10: 850-860, 2009. 
25. Colcombe SJ, Erickson KI, Raz N, Webb AG, Cohen NJ, McAuley E and Kramer AF: Aerobic fitness reduces brain tissue loss in aging humans. J Gerontol A Biol Sci Med Sci 58: 176-180, 2003.

26. Kramer AF, Colcombe SJ, McAuley E, Scalf PE and Erickson KI: Fitness, aging and neurocognitive function. Neurobiol Aging 26 (Suppl 1): S124-S127, 2005.

27. Erickson KI, Colcombe SJ, Elavsky S, McAuley E, Korol DL, Scalf PE and Kramer AF: Interactive effects of fitness and hormone treatment on brain health in postmenopausal women. Neurobiol Aging 28: 179-185, 2007.

28. Rodrigues MA, Verdile G, Foster JK, Hogervorst E, Joesbury K, Dhaliwal S, Corder EH, Laws SM, Hone E, Prince R, et al: Gonadotropins and cognition in older women. J Alzheimers Dis 13: 267-274, 2008.

29. Lee MC, Okamoto M, Liu YF, Inoue K, Matsui T, Nogami H and Soya H: Voluntary resistance running with short distance enhances spatial memory related to hippocampal BDNF signaling. J Appl Physiol (1985) 113: 1260-1266, 2012.

30. Quirié A, Hervieu M, Garnier P, Demougeot C, Mossiat C, Bertrand N, Martin A, Marie C and Prigent-Tessier A: Comparative effect of treadmill exercise on mature BDNF production in control versus stroke rats. PLoS One 7: e44218, 2012.

31. Gomez-Pinilla F and Hillman C: The influence of exercise on cognitive abilities. Compr Physiol 3: 403-428, 2013.

32. Schefer V and Talan MI: Oxygen consumption in adult and AGED C57BL/6J mice during acute treadmill exercise of different intensity. Exp Gerontol 31: 387-392, 1996.

33. Kritzer MF and Kohama SG: Ovarian hormones differentially influence immunoreactivity for dopamine beta-hydroxylase, choline acetyltransferase, and serotonin in the dorsolateral prefrontal cortex of adult rhesus monkeys. J Comp Neurol 409: 438-451, 1999.

34. Numakawa T, Yokomaku D, Richards M, Hori H, Adachi N and Kunugi H: Functional interactions between steroid hormones and neurotrophin BDNF. World J Biol Chem 1: 133-143, 2010.

35. Seidah NG, Benjannet S, Pareek S, Chrétien M and Murphy RA: Cellular processing of the neurotrophin precursors of NT3 and BDNF by the mammalian proprotein convertases. FEBS Lett 379: 247-250, 1996.

36. Lu B, Pang PT and Woo NH: The yin and yang of neurotrophin action. Nat Rev Neurosci 6: 603-614, 2005.

37. Lessmann V, Gottmann K and Malcangio M: Neurotrophin secretion: Current facts and future prospects. Prog Neurobiol 69: 341-374, 2003
38. Mowla SJ, Pareek S, Farhadi HF, Petrecca K, Fawcett JP, Seidah NG, Morris SJ, Sossin WS and Murphy RA: Differential sorting of nerve growth factor and brain-derived neurotrophic factor in hippocampal neurons. J Neurosci 19: 2069-2080, 1999.

39. Greenberg ME, Xu B, Lu B and Hempstead BL: New insights in the biology of BDNF synthesis and release: Implications in CNS function. J Neurosci 29: 12764-12767, 2009.

40. Pang PT, Teng HK, Zaitsev E, Woo NT, Sakata K, Zhen S, Teng KK, Yung WH, Hempstead BL and Lu B: Cleavage of proBDNF by tPA/plasmin is essential for long-term hippocampal plasticity. Science 306: 487-491, 2004.

41. Waterhouse EG and $\mathrm{Xu} \mathrm{B}$ : New insights into the role of brain-derived neurotrophic factor in synaptic plasticity. Mol Cell Neurosci 42: 81-89, 2009

42. Cohen-Cory S, Kidane AH, Shirkey NJ and Marshak S Brain-derived neurotrophic factor and the development of structural neuronal connectivity. Dev Neurobiol 70: 271-288, 2010.

43. Cowansage KK, LeDoux JE and Monfils MH: Brain-derived neurotrophic factor: A dynamic gatekeeper of neural plasticity. Curr Mol Pharmacol 3: 12-29, 2010.

44. Binder DK and Scharfman HE: Brain-derived neurotrophic factor. Growth Factors 22: 123-131, 2004.

45. Woo NH, Teng HK, Siao CJ, Chiaruttini C, Pang PT, Milner TA, Hempstead BL and Lu B: Activation of p75NTR by proBDNF facilitates hippocampal long-term depression. Nat Neurosci 8: 1069-1077, 2005.

46. Srivastava DP, Woolfrey KM and Evans PD: Mechanisms underlying the interactions between rapid estrogenic and BDNF control of synaptic connectivity. Neuroscience 239: 17-33, 2013.

47. Harrington AW, Kim JY and Yoon SO: Activation of Rac GTPase by $\mathrm{p} 75$ is necessary for c-jun $\mathrm{N}$-terminal kinase-mediated apoptosis. J Neurosci 22: 156-166, 2002.

48. Becker EB, Howell J, Kodama Y, Barker PA and Bonni A: Characterization of the c-Jun N-terminal kinase-BimEL signaling pathway in neuronal apoptosis. J Neurosci 24: 8762-8770, 2004.

49. Bhakar AL, Howell JL, Paul CE, Salehi AH, Becker EB, Said F, Bonni A and Barker PA: Apoptosis induced by p75NTR overexpression requires Jun kinase-dependent phosphorylation of Bad. J Neuroscience 23: 11373-11381, 2003.

50. Sierksma AS, Vanmierlo T, De Vry J, Raijmakers ME, Steinbusch HW, van den Hove DL and Prickaerts J: Effects of prenatal stress exposure on soluble $\mathrm{A} \beta$ and brain-derived neurotrophic factor signaling in male and female APPswe/PS1dE9 mice. Neurochem Int 61: 697-701, 2012.

51. Johansson BB and Ohlsson AL: Environment, social interaction, and physical activity as determinants of functional outcome after cerebral infarction in the rat. Exp Neurol 139: 322-327, 1996. 